\title{
Research on Power Stability Control of Semiconductor Laser
}

\author{
Qian Guo ${ }^{1}$,Xiaodong Zhang ${ }^{2, a}$,Fengnian Tian $^{3}$ \\ ${ }^{1}$ School of Mechanical and Electrical Engineering,Xi' an Polytechnic University,710048 Xi'an,Shaanxi,China \\ ${ }^{2}$ School of Mechanical Engineering,Xi'an Jiaotong University, 710049 Xi'an,Shaanxi,China \\ ${ }^{3}$ School of Aeronautics and Astronautics,Huazhong University of Science and Technology,430074 Wuhan,Hubei,China
}

\begin{abstract}
In order to guarantee the stable emission power and good working environment of semiconductor laser,a new hardware circuit is selected and designed to realize automatic optical power control,in which a negative feedback operation amplifier circuit is adopted to form the constant current source, and an optoelectronic feedback to realize closed loop control in this paper.And then,the corresponding steady control circuit is also designed,so that the stable power can be output.The experimental results show that the stability of laser power is better than $0.74 \%$ when the system works.
\end{abstract}

\section{Introduction}

Semiconductor lasers are the most practical and important type of lasers in the field of optoelectronics ${ }^{[1]}$. However,as the semiconductor laser is a diode structure ${ }^{[2]}$,its performance will change with the extension of service time or affected by some other components in the circuit ${ }^{[3]}$. At the same time,the semiconductor laser is a non-linear device,which is greatly influenced by temperature and other factors ${ }^{[4]}$. The change of working temperature will affect the output wavelength,threshold current and output power of the semiconductor laser.In practical application,the performance of semiconductor lasers and the stability of output power will directly affect the result of system operation. Therefore,LD needs a special driver to compensate the influence of various factors and stabilize the transmitting power.In this regard,some scholars have carried out research,for example,Hyeoungho Bae et al. from the university of California used the negative feedback principle to control the driving current of the semiconductor laser to complete automatic power control,which ensured the stability of the output wavelength and coherent length of the semiconductor laser ${ }^{[5]}$.E.Sackinger and y.Ota of bell experiment in the United States use automatic power control circuit and performance detection circuit to complete laser drive,with low power consumption and stable output power.Zhou zhen et al.of Harbin University of science and technology used constant current source control and automatic power control to drive semiconductor lasers, which could guarantee the power stability better than $1 \%$.

A low power LD drive circuit for semiconductor laser automatic power control is introduced in this
paper.The circuit is applied to direct modulation of semiconductor lasers and good control effect is achieved.

\section{Power stability compensation control principle of semiconductor laser}

\subsection{Internal structure of semiconductor laser}

A common semiconductor laser is a laser Diode LD encapsulated with a photodiode(Photo-bond,PD), whose internal structure is shown in Figure $1^{[6]}$.As the light emitted by LD includes forward laser and back laser,the wavelength and light power of forward laser and back laser are identical,so the forward light power of LD can be obtained by measuring the back laser.

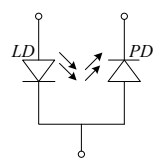

Figure1.The internal structure diagram of laser

\subsection{P-I characteristics of semiconductor lasers}

An important characteristic of semiconductor lasers is the power-current characteristic,also known as the P-I characteristic,as shown in figure ${ }^{[7]} . \mathrm{P}$ represents the optical power $(\mathrm{mW})$ output of the laser,and I represents the injection current $(\mathrm{mA})$ of the laser.P-I characteristics are the changes of the output optical power of the laser with the injection current.

As can be from Figure2, when the injection current increases to a certain value,the output optical power

\footnotetext{
a Corresponding author: xdzhang@mail.xjtu.edu.cn
} 
increases sharply,and the luminescence characteristics also change greatly,resulting in laser oscillations. This current is called the threshold current,which is represented by $\mathrm{I}_{\mathrm{th}}$.It is the current corresponding to the inflexion of P-I curve.

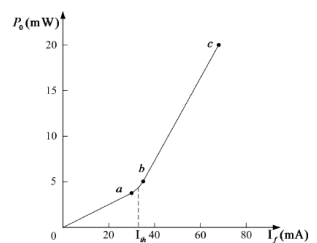

Figure2.Semiconductor laser P-I characteristic curve

\subsection{Power stability control principle of semiconductor laser}

The common semiconductor lasers are often excited by junction current injection. When the injection current is larger than the threshold current,the radiation power increases rapidly with the increase of current.Therefore,the output optical power can be adjusted by changing the injection current of the semiconductor laser.There are many methods for Semiconductor laser automatic control:one is constant current control (ACC), the other is constant power control (APC), and the third is constant voltage control (AVC).This method is also called automatic control of optical power.This system adopts APC control.Its structure diagram is shown in figure 3.

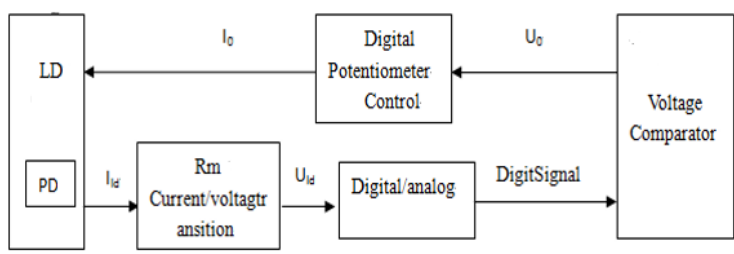

Figure3.Optical power stability control schematic

In Figure3,PD detected a part of optical power that can reflect the change of output optical power linearly from the back-facing laser of the laser chip,which is transformed into an electrical signal through photoelectric conversion. The signal is compared with the reference voltage and amplified by the amplifier circuit to control the $\mathrm{dc}$ bias current of the laser,so that the output optical power of LD can be stabilized by changing the magnitude of the bias current.

\section{Design on power stability control circuit of semiconductor laser}

APC of automatic control circuit of optical power mainly includes comparison amplifier module,constant current source module and optical power feedback module.

\subsection{Compare and enlarge module}

In order to obtain high precision voltage signal,a comparison amplifier is designed by using
INA114.INA114 is set voltage signal input,INA114 by photoelectric diode reverse input $\mathrm{PD}$ and feedback module testing and feedback voltage signal,INA114 then the system Settings of the forward and reverse voltage at the input voltage difference value of amplification,among them, the $\mathrm{RG}$ resistance is used to adjust magnification $G$,the relationship between available under type said:

$$
\begin{aligned}
& G=1+\left(50 K \Omega / R_{G}\right) \\
& \mathrm{U}_{1}=\mathrm{G}\left(\mathrm{U}_{+}-\mathrm{U}_{-}\right)+\mathrm{U}_{3}
\end{aligned}
$$

In formula(1) and formula(2), $G$ is the amplification coefficient of the amplifier,which is the external amplification resistance.The amplification factor of output voltage can be controlled by changing the size.Is the reference voltage, is the optical power feedback voltage, is the comparison amplifier output voltage.

\subsection{Constant current source module}

The current flowing through R0 is the driving current of LD.Where,OPA547 and OPA177 are voltage followers. The voltage follower constituted by OPA177 is used to send the voltage on LD to the Ref pin of INA114,namely V3=V4.At this time, the current I flowing through LD can be obtained by the following formula:

$$
I=(V 2-V 4) / R f=G(V+-V-) / R f
$$

Under normal conditions,LD works at the set point. The driving current I through LD and the output optical power of LD maintain a stable balance.

\subsection{Negative feedback module}

MAX4008 and OPA320 are used to form optical power feedback circuit.MAX4008 is a high-precision current detection chip,which is specially used to detect the current of PD or PIN photoelectric detector in fiber applications.Its REF PIN is the reference current input PIN, and the OUT PIN is the detection voltage output PIN.The relation between the output voltage and the reference current of MAX4008 is:

$$
\begin{aligned}
& U_{\text {Out }}(m V)=I_{R e f}(\mu A) \\
& U_{5}(m V)=\mathrm{I}_{1}(\mu \mathrm{A})
\end{aligned}
$$

The voltage fed back by MAX4008 is amplified through OPA320 operational amplifier,then the feedback voltage is:

$$
U_{-}=U_{5}\left(1+R_{f} / R\right)
$$

Where, is the scale magnification coefficient,and can be changed according to the parameters of LD and PD in practical application.

\subsection{System circuit of optical power stability control (APC)}

The circuit schematic diagram is shown in Figure 4. When the output optical power of the laser increases and the optical current detected by photodiode PD increases, the feedback voltage loaded at the inverted input terminal of the operational amplifier increases.Since the reference voltage $U_{+}$value of the 
operational amplifier is unchanged at the same direction of the input terminal,the difference between the two will decrease,resulting in the decrease of the output voltage U1 of the operational amplifier.In this way,the current flowing through R0 decreases,so the injection current of the semiconductor laser decreases.According to the P-I characteristic curve of the semiconductor laser,the injection current decreases, which will reduce the output power of the laser.On the contrary, when the optical power output of the laser decreases, the optical current flowing through PD decreases, and the feedback voltage decreases, the output voltage of the operational amplifier increases.In this way, the driving current flowing through the semiconductor laser increases, thereby regulating the increase of LD output power.APC circuit can balance the driving current and output power and realize automatic power control.

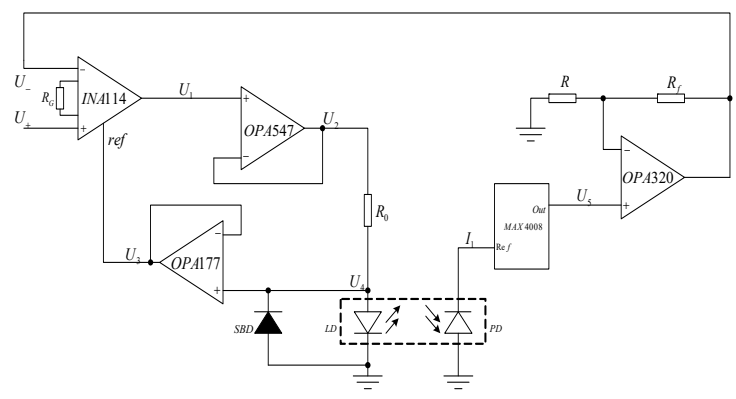

Figure4.Optical power stability control system diagram

The semiconductor laser is a very fragile element,vulnerable to electrostatic or reverse voltage shock,so it is necessary to protect the circuit against LD damage.In this paper,a schottky diode is used in parallel with LD to protect LD.

\section{Control circuit characteristic analysis and modelling simulation}

\subsection{Analysis of circuit characteristics of each module}

Automatic power control system composed of operational amplifierI,II,controlled object,feedback,operational amplifier unit and other parts.

The transfer function of each component is listed separately.

(1)Operational amplifierI: OPA114 for proportional amplifier,its input (reference voltage $\mathrm{U}+\mathrm{U}$ - and feedback voltage deviation and amplified,the transfer function for operational amplifier magnification $\mathrm{G} 1=\mathrm{G}=1$.

(2)Operational amplifierII:OPA547 composition proportion,the relationship between the output voltage and input voltage for $\mathrm{U} 2=(\mathrm{R} 2 / \mathrm{R} 1) \mathrm{U} 1$, its transfer function $\mathrm{G}=2 \mathrm{R} 2 / \mathrm{R} 1=\mathrm{Kp}$.

(3)Controlled object:according to the reference, the transfer function of the laser output to the photoelectric detector can be tested by signal source sweep frequency,and the relationship between amplitude and phase of the link can be tested,and then the transfer function can be deduced.According to the test results, the laser is a first-order inertial link in the optical intensity control system, and its transfer function is:

$$
G_{3}(S)=\frac{K}{T_{S}+1}
$$

The transfer function of the laser element is identified by using the tangent method.Experiment,the first by manual operation to make the process work under the condition of the steady state of test required,stable operation after a period of time, the input of the process of rapid change,record the change of the process of the output curve,after a period of time,the process into a new steady state,the end of the experiment,the record of step response curve is process. The tangent method makes the tangent line at the inflection point of the curve, and intersects at the horizontal and vertical axes respectively.The difference between the steady-state value and the input value of the system is $\mathrm{K}$. The projection of the intersecting line on the horizontal axis is T.The laser output step response curve is shown in Figure 5.

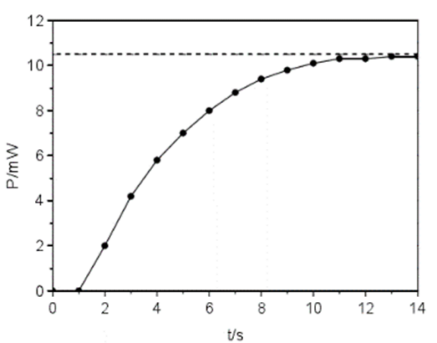

Figure5.Laser output step response curve

According to figure $5, \mathrm{~K}=10.5$ and $\mathrm{T}=7-1=6$.Then, the transfer function of LD of the controlled object identified by the system is:

$$
G_{3}(S)=\frac{10.5}{6 S+1}
$$

According to the control theory,the forward channel of the automatic power control loop is a series of two control links, and its transfer function is:

$$
G(S)=G_{2}(S) G_{3}(S)=\frac{1.75 K_{P}}{S+0.167}
$$

(4)Feedback unit:the photoelectric feedback unit is composed of photodiode,current and voltage conversion,and phase proportional amplifier.The unit can be approximated as a first-order inertial link,and its transfer function is:

$$
H(S)=\frac{K}{T_{S}+1}
$$

Where, $\mathrm{T}$ is the time constant and $\mathrm{K}$ is the gain coefficient.Similarly, the transfer function of the feedback unit is identified by the tangent method through step response modeling.

According to the above formula, $\mathrm{K}=0.5, \mathrm{~T}=5$ $1=4$.Then, the transfer function of LD of the controlled object identified by the system is:

$$
H(S)=\frac{0.5}{4 S+1}
$$

\subsection{Transfer characteristics analysis of stability control system}


The open-loop transfer function of the automatic power control loop is the product of the forward channel transfer function and the feedback unit transfer function.

That is:

$$
G_{0}(S)=G(S) H(S)=\frac{0.2188}{S_{2}+0.4167 S+0.0417} K_{P}
$$

Its closed-loop transfer function is:

$$
(S)=\frac{G_{S}}{1+G(S) H(S)}=\frac{1.75 S+0.4375}{S_{2}+0.4167 S+0.0417+0.2188 K_{P}} K_{P}
$$

Based on the transfer function of each control unit,the automatic power control system can be simulated.The simulation will be in the model

$\mathrm{K} \mathrm{P}$ is set to $0.35,0.19$ and 0.15 respectively, and the unit step response curve when proportional $\mathrm{P}$ is controlled by the oscilloscope is obtained, as shown in Figure6.

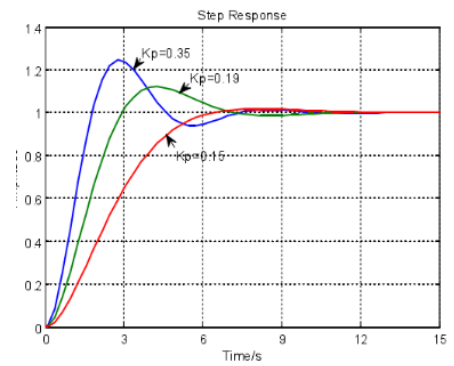

Figure6.The unit step response curve for proportional $\mathrm{P}$ control

\section{Experimental verification}

Under the room temperature is $20^{\circ} \mathrm{C}$, the semiconductor laser drive current stability experiment:by measuring the sampling resistor $\mathrm{R}$ at both ends of the voltage injection current can be calculated.Then, according to the working principle of the circuit and the linear relation between measuring voltage and driving current,the variation curve of driving current is obtained,as shown in Figure 7.The experimental semiconductor laser has a wavelength of $650 \mathrm{~nm}$ and a power rating of $15 \mathrm{~mW}$.

As can be seen from the variation curve of the driving current in Figure6, the driving current fluctuates within the range of $80.233 \sim 80.246$, with the maximum variation of $0.013 \mathrm{~mA}$ and the current stability of $0.013 \%$,meeting the design requirements.

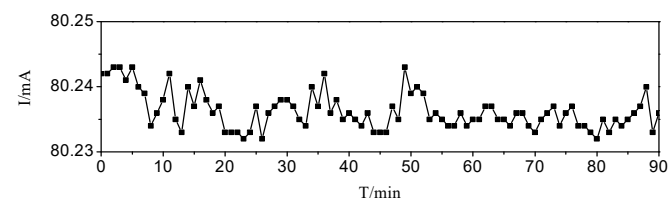

Figure7.Open loop work curve of the constant current source in the APC

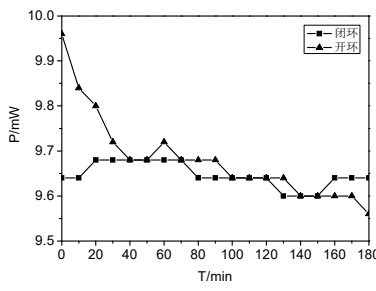

Figure8. The open loop and closed loop power curve of $\mathrm{LD}$ in the APC

The output power of the semiconductor laser stabilized control system is tested under two conditions of open and closed loop.Experimental conditions: the drive current is 80 ma,temperature is $20^{\circ} \mathrm{C}$ at room temperature,

preheat circuit $15 \mathrm{~min}$, and respectively to reverse proportion amplifier input ground,reverse the input circuit to 3 hours,sampling system,the experimental measured curve as shown in Figure 8.It can be seen from the figure that the output power decreases gradually and fluctuates greatly when the loop is opened.When closed,the output power is stable and the fluctuation range is controlled within $0.1 \mathrm{mw}$.

\section{Conclusions}

Aiming at the practical application of small power semiconductor lasers,an automatic control method of optical power is proposed to make them working stably, and the LD optical power stably control circuit is also designed in this paper.Based on the principle of photoelectric negative feedback control,the circuit collects and calculates the output optical power of the semiconductor laser,dynamically adjusts the size of LD injection current and compensates the change of output optical power,so as to achieve the work requirement of the stability of the optical power of the semiconductor laser,which can be widely used in practical engineering.

\section{Acknowledgement}

The authors are grateful for the support provided by the Natural Science Foundation Project of Shaanxi province and the science and technology innovation project of $\mathrm{Xi}$ 'an Science and Technology Bureau.

1) Natural science foundation project of shaanxi province "Research On High Order Modulation of Wireless Optical Orthogonal Frequency Division Multiplexing System".

2)The science and technology plan of xi 'an city (201805030YD8CG14 (12)).

\section{References}

1. Ke xizheng, deng lijun. Wireless optical communication [M].Beijing: science press,2016(10).

2. Ke xizheng,Chen Juan. Space-time coding theory in wireless optical MIMO system [M].Beijing: science press,2014(4). 
3. Ke xizheng,Yin zhiyun.Coding theory in wireless laser communication system [M].Beijing:science press,2009(9).

4. Ke xizheng,Zhao li, Yin zhiyun,Hu shuqiao. Experimental study on error control in wireless laser communication [J].Journal of electronic measurement and instrumentation,2009,06:18-23.

5. Ke xizheng,Yin zhiyun,Yang lihong.Modulation scheme of light PPM polarization in atmospheric laser communication and its key technologies [J].Semiconductoroptoelectronics,2007,04:553$555+560$.

6. Z.Zhou,F.Wang,Z.Yang,X.Teng.The study of optical power control for driving circuit using the 650 nanometer low power semiconductor laser[C].IEEE International Conference on Measurement,

Information and Control,2013,1:222-224.

7. Chen ying.Control system development of optical fiber output stable laser light source [M].Jinan university.2013.05. 